\title{
Design and Control of a Powered Knee Orthosis for Gait Assistance
}

\author{
Hao Ma ${ }^{1}$, Wai-Yin Lai ${ }^{1}$, Wei-Hsin Liao ${ }^{1}$, Daniel Tik-Pui Fong ${ }^{2}$, and Kai-Ming Chan ${ }^{2}$
}

\begin{abstract}
A powered knee orthosis named Assistive Knee Brace (AKB) is designed for the elderly and patients with knee dysfunctions to gain external support at their knee joints during gait. Safety, light-weight and user-friendly interface are the emphasis of system design. In the assistive controller design, gait analysis method based on Finite State Machine is developed for gait pattern estimation, with state estimated through fuzzy inference. Based on the gait pattern estimated online, reference gait pattern is generated as assistive control input of the powered knee orthosis to adjust the wearer's abnormal motion with torque support to track the normal pattern. To verify the assistive function of $A K B$, gait experiments under control are conducted on healthy subjects simulating certain limited mobility. Results show that with proper assistive torque from the AKB, wearers can perform motions in good patterns.
\end{abstract}

\section{INTRODUCTION}

The impaired mobility problem has badly affected the life quality of an increasing population of the elderly and patients suffering from some diseases. They have strong demands in devices that can provide assistive functions to help their gait rehabilitation and daily walking. Compared to medical passive orthosis, powered lower limb orthosis with appropriate control would provide more assistive, flexible and comfortable support to human daily motions.

Several powered lower limb orthoses have been developed, such as the Hybrid Assistive Limb by Sankai [1], Vanderbilt Exoskeleton by Goldfarb [2], ReWalk by Goffer [3], and eLeg by Ekso Bionics [4], to provide assistive functions at the wearer's lower limb joints in the sagittal plane. These devices were mainly designed for paraplegic gait assistance. However, for the elderly and less severe patients with dysfunction mainly in knee joint(s), such as those cannot bend knee or weak in muscle strength around knee, they still have certain disordered mobility. Wearing a whole set of lower limb orthosis with redundant actuators could be an annoying task, and may further have negative effect in the rehabilitation of joint functions. Therefore, in this study we would like to focus on the users with knee dysfunction to design a powered knee orthosis (PKO) named Assistive Knee Brace (AKB).

The basic features of AKB design include light-weighted, comfortable connection, safe and intelligent controller to provide assistive functions. Since the knee orthosis is just attached between thigh and shank, the weight will be supported by the hip joint in the swing phase. In order to comfortably maintain normal loading for the hip joint, weight becomes a critical issue in the design. Therefore, we tried to reduce the structure and in the meantime ensure

H. Ma, W. Y. Lai, and W. H. Liao are with the Department of Mechanical and Automation Engineering, The Chinese University of Hong Kong, Hong Kong SAR, China. \{hma, wylai2,whliao\}@mae.cuhk.edu.hk. D. T. P. Fong and K. M. Chan are with the Department of Orthopaedics \& Traumatology, The Chinese University of Hong Kong, Hong Kong SAR, China.dfong@ort.cuhk.edu.hk, kaimingchan@cuhk.edu.hk. safety and keep enough torque generation capability for normal usage.

Along with the overall mechatronic design of $\mathrm{AKB}$ system, an integrated gait analysis and control method is developed for assisting the wearer's gait on the level ground. The target users with disordered gait pattern are expected to regain normal gait pattern with assistive torque from the AKB under control. Due to interaction between human body and $\mathrm{AKB}$, the gait pattern under assistive control may be different from normal pattern during the rehabilitation process. Thus, online gait pattern analysis is focused in the controller design presented here.

For the analysis of both normal and disordered gait patterns, states representing different human body postures and foot-ground contact forms are defined and the gait cycles are described in a Finite State Machine (FSM) as transitions between sequential dominant states. Fuzzy logic inference is applied for a continuous and smooth estimation of state. Wearer's individual gait pattern is obtained by analyzing the state sequences. Then an adaptive reference knee angle curve is generated based on the actual gait pattern for comfortable and safe assistive control.

The content of this paper is arranged as follows. Section II gives out the AKB system design including mechanical structure, electrical design and sensing system. Section III introduces human gait analysis methods we propose for state and gait pattern estimation. Section IV provides the AKB controller design for assistive function. Section $\mathrm{V}$ presents the experiments and obtained results. Section VI is the conclusion and future perspective.

\section{SYSTEM DESIGN}

The AKB system design is shown in Fig. 1 with three major aspects: mechanical structure, electrical system, and control program. This section mainly introduces the hardware of the AKB system in order to achieve a light-weighted, safe, and user-friendly design.

\section{A. Mechanical Design}

The AKB mechanical structure in right knee configuration contains three main parts: knee orthosis,

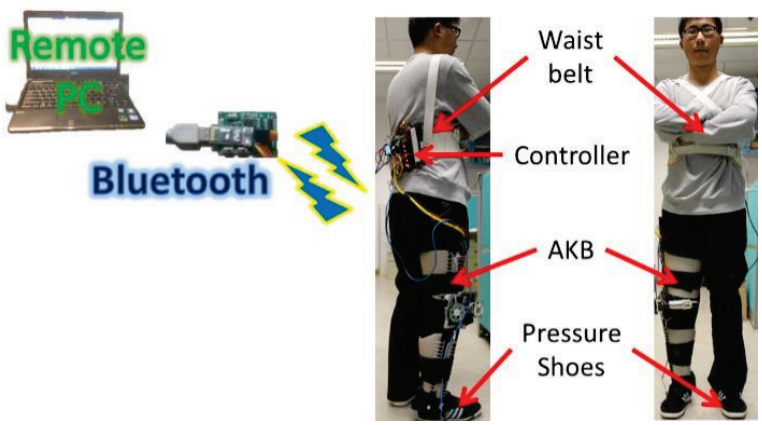

Figure 1. Schematic of AKB system 


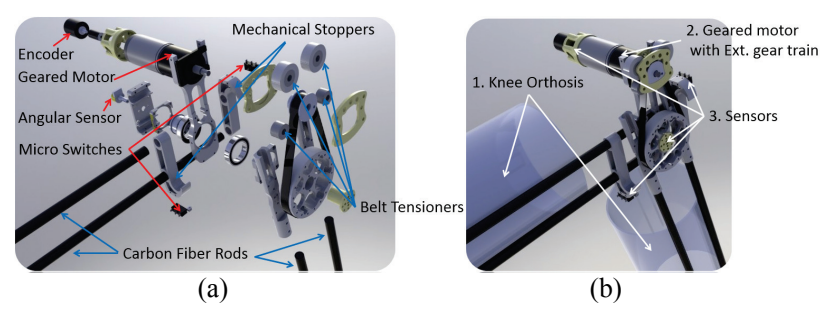

Figure 2. (a) Exploded view, and (b) assembly of the AKB mechanical structure

geared motor with an external gear train, and sensors system, as shown in Fig. 2. They are assembled to the aluminum structure and carbon fiber rods for making a rigid and light-weight frame.

The knee orthosis is custom-made of Very Low Density Polyethylene (VLDPE) thermal plastic to fit the shape of human limbs. The geared motor, weighting $580 \mathrm{~g}$ yet providing a $16 \mathrm{Nm}$ stall torque, has a torque constant 0.85 $\mathrm{Nm} / \mathrm{A}$. A pair of aluminum sprocket (15T:48T) with gear ratio of 3.2:1 is realized by using a timing belt system, so that a high transmitting efficiency about $\eta=0.98$ could be achieved [5]. This configuration can make the max torque to reach up to $45 \mathrm{Nm}$. With large and firm contact surface, just $10 \mathrm{~mm}$ is enough to take over the torsion in the configuration. It reduces the motor spacing in the top view projection. A 50CPR encoder is mounted at the rear shaft of the motor and an external angular sensor is placed at the driven sprockets for reading the absolute knee angle. Two micro contact switches are placed at the terminals on the mechanical stoppers to detect the limit cases of the rotation motion.

Safety is also one key issue in the AKB design. Besides the redundancy of sensors for software protection in control, the mechanical stoppers work in a pair to limit the knee angle working range within $0^{\circ}-120^{\circ}$. Under two extreme motions in flexing and extension, the factor of safety (FOS) is designed to have larger than 3 under $60 \mathrm{Nm}$ loading. FOS is also considered for all other parts, which are all over 2. The stoppers are strong enough to prevent the user from injury in case accident happens.

The net weight of the AKB is $2 \mathrm{~kg}$ including $800 \mathrm{~g}$ VLDPE braces, which make it so far the lightest power lower limb orthosis to the best of our knowledge. Additional $1 \mathrm{~kg}$ of batteries and controller board are put onto the waist belt apart from the AKB to decrease the loading. The light weight characteristic would bring out the power-saving merit since less actuating and mechanical structure are required compared to other powered orthoses and exoskeletons.

\section{B. Electrical Design}

Based on the torque constant and the maximum required torque, the estimated maximum driving current and voltage would be $19 \mathrm{~A}$ and $16 \mathrm{~V}$ respectively. To deal with such a high power control, besides using a high current motor driving unit, a radiator is also needed to maintain the IC temperature in normal level during the driving. We fabricated the controller that fits the AKB input/output as shown in Fig. 3.

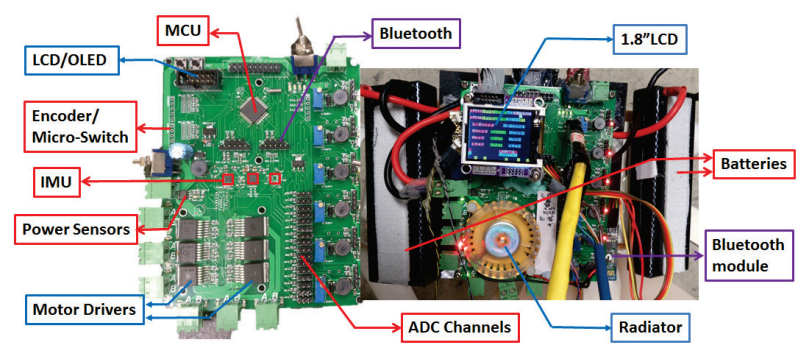

Figure 3. Photos of the PCB and Power-on PCB controller. (Red and blue lines are the input and output of the system, respectively)

The controller is able to use 1-2 pieces of lithium polymer battery, which has $7.4 \mathrm{~V}$ with $5.1 \mathrm{AH}$ and weight $300 \mathrm{~g}$ each. The operating duration is estimated more than 1 hour in normal use. Bluetooth-to-UART is adopted for communication of both remote control and data logging in PC. Embedded power sensors are used for checking the input current and voltage for measuring the motor output torque and power.

Ground reaction force (GRF) is important information associated with human gait that indicates the foot-ground contact conditions. In this research, gait patterns are analyzed based on the pattern of GRFs. Similar to some other researches $[1,6-8]$, pressure shoe pads are designed to obtain GRFs and plantar pressure distribution in both feet, with 4 Force Sensing Resistors (FSRs) installed at specific locations as shown in Fig. 4. FSR sensor can sample the pressure value at these locations.

\section{HUMAN GAIT ANALYSIS}

Gait analysis is an important way to study and evaluate gait pattern, and further help in the assistive control design. In this study, we take advantage of online gait pattern analysis results, especially focus on the pattern of GRF, to have an adaptive and comfortable gait assistance design. This section will introduce the Finite State Machine based human gait analysis methods developed for AKB control.

\section{A. Finite State Machine Definition}

Human gait is basically continuous periodic motions. However, from the perspective of alternating movements of both legs, we can consider the whole motion cycle consists of transitions between several discrete states [9]. Different state represents different body configuration and contact form with ground, and therefore within each state human motion state represents different body configuration and contact form with ground, and therefore within each state human motion follows respective kinematic and dynamic equations. Based on this idea, Finite State Machine (FSM)

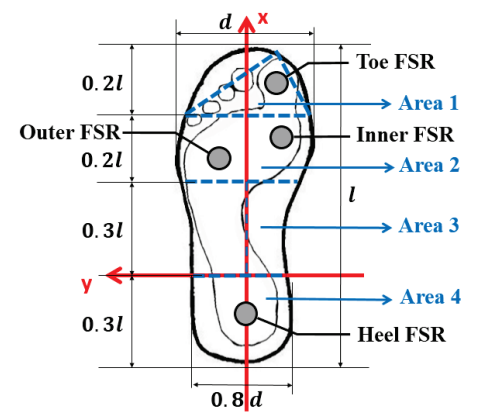

Figure 4. Pressure shoe pad with 4 FSR sensors 
TABLE I. STATE DEFINITION IN FINITE STATE MACHINE

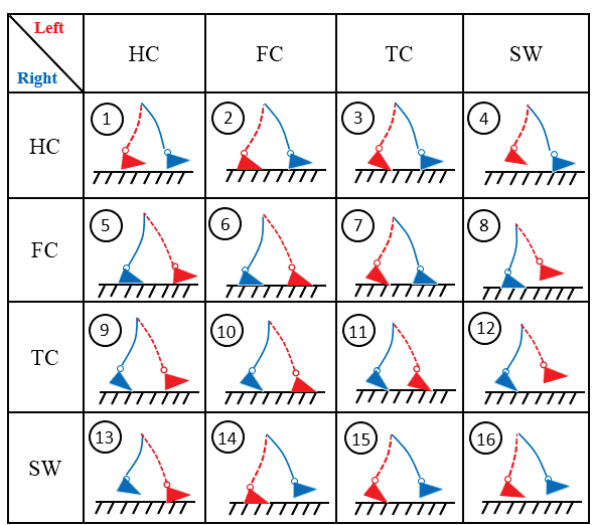

based gait analysis method is proposed and human gait cycle is described as a series of transitions between each state.

If we consider the contact form with ground for single leg, we can divide it into 4 cases:

1) Heel Contact (HC): only heel contacts ground;

2) Full Contact (FC): heel and front contact ground;

3) Toe Contact (TC): only front/toe part contacts ground;

4) Swing (SW): the entire foot leaves ground;

Full set of states are defined by the combination of contact forms of both legs shown in Table I. Foot events including Heel Strike (HS), Front Contact (FC), Heel Off (HO), and Toe Off (TO) in both legs are transition conditions that drive state transfers from one to another during human gait.

\section{B. Estimation of State within Gait Cycle}

Foot-ground contact forms could be detected by monitoring GRF distributions in each foot. Traditional methods detect stages of gait by setting thresholds to the pressure sensor values [1], which are discrete and sensitive around decision boundary. Kong et al. have applied fuzzy logic in the stage detection of single leg case [8]. With FSM method, we can consider state transitions in both legs, which could provide more information in a systematic way in the gait analysis compared to traditional methods. Fuzzy logic inference is used for mapping input sampled force of each.

FSR sensor to output probability vector of contact forms, so that we could obtain continuous and smooth estimation of states, which will contribute to a continuous estimation of COG, joint positions and torques in kinematics and dynamics calculation. Fig. 5 shows the overall structure of fuzzy logic inference. The input force value $f$ of each FSR goes through fuzzification with membership function (1) and (2). The fuzzy logic inference rules are set according to Table II, with "L" stands for Large and "S" stands for Small.

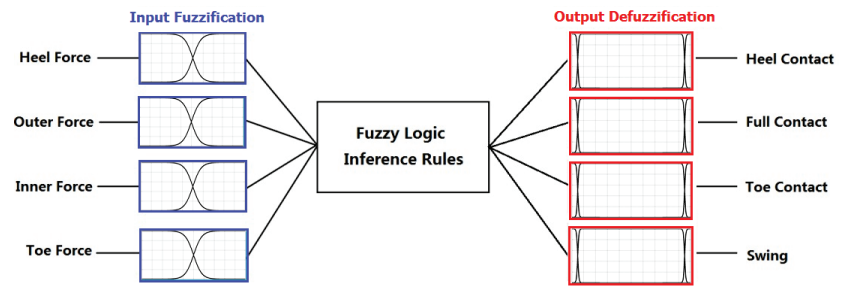

Figure 5. Contact form detection based on fuzzy logic
TABLE II. FUZZY INFERENCE RULES

\begin{tabular}{|c|c|c|c|c|c|c|c|c|}
\hline \multirow{2}{*}{ Rules } & \multicolumn{4}{|c|}{ Ground Reaction Force } & \multicolumn{4}{|c|}{ Contact Condition } \\
\hline & Heel & Outer & Inner & Toe & $\mathrm{HC}$ & FC & $\mathrm{TC}$ & SW \\
\hline 1 & $\mathrm{~L}$ & $\mathrm{~S}$ & $\mathrm{~S}$ & $\mathrm{~S}$ & $\mathrm{~L}$ & $\mathrm{~S}$ & $\mathrm{~S}$ & $\mathrm{~S}$ \\
\hline 2 & $\mathrm{~L}$ & $\mathrm{~L}$ & $\mathrm{~N} / \mathrm{A}$ & $\mathrm{N} / \mathrm{A}$ & $\mathrm{S}$ & $\mathrm{L}$ & $\mathrm{S}$ & $\mathrm{S}$ \\
\hline 3 & $\mathrm{~S}$ & \multicolumn{3}{|c|}{ At least one is Large } & $\mathrm{S}$ & $\mathrm{S}$ & $\mathrm{L}$ & $\mathrm{S}$ \\
\hline 4 & $\mathrm{~S}$ & $\mathrm{~S}$ & $\mathrm{~S}$ & $\mathrm{~S}$ & $\mathrm{~S}$ & $\mathrm{~S}$ & $\mathrm{~S}$ & $\mathrm{~L}$ \\
\hline
\end{tabular}

The output after centroid defuzzification of functions (3) to (5) are the probabilities $P$ of the current condition belonging to each contact form. Then the contact form vector is defined as the vector $=\left(P_{H C} P_{F C} P_{T C} P_{S W}\right)$ after normalization.

Input membership functions:

$$
\begin{gathered}
\mu^{\text {Large }}(f)=\frac{1}{1+\left(\frac{f_{0}}{f}\right)^{\lambda}} \text { with } f_{0}=1.5 N, \lambda=2.5 \\
\mu^{\text {Small }}(f)=1-\mu^{\text {Large }}(f)
\end{gathered}
$$

Output membership functions:

$$
\begin{gathered}
\mu^{\text {Large }}(x)=\frac{1}{1+e^{-v\left(x-x_{0}\right)}} \text { with } v=200, x_{0}=0.95 \\
\mu^{\text {Small }}(x)=\frac{1}{1+e^{v\left(1-x_{0}-x\right)}} \text { with } v=200, x_{0}=0.95 \\
\mu^{\text {Middle }}(x)=1-\mu^{\text {Large }}(x)-\mu^{\text {Small }}(x)
\end{gathered}
$$

Then state matrix is further defined as the product of the contact form vector of both feet by (6). The normalized 4X4 matrix contains the probability $P_{i}$ of all corresponding state $i$ defined in Table II. For computational convenience, we only consider states with probabilities larger than 0.1 as effective states, and re-normalize the state matrix with only effective states.

$$
\mu_{\text {State }}=\mu_{\text {Left }}^{T} \cdot \mu_{\text {Right }}=\left[\begin{array}{cccc}
P_{1} & P_{5} & P_{9} & P_{13} \\
P_{2} & P_{6} & P_{10} & P_{14} \\
P_{3} & P_{7} & P_{11} & P_{15} \\
P_{4} & P_{8} & P_{12} & P_{16}
\end{array}\right]
$$

After this process, we could still approximately maintain the continuity of state transitions. On the other hand, discrete state transitions could also be obtained by discretization method to take the state with maximal probability as the current dominant state.

\section{State Transitions within Gait Cycle}

The developed FSM based analysis method is applied in the analysis of gait in level ground. An example of state estimation results within three walking cycles of level walking of a healthy subject are shown here.

The detected contact form of the right leg is given in Fig. 6 , with the dominant contact form shown at the bottom. With fuzzy inference, it could give out reliable contact form detection even though there is a small variance in the GRF. State transition estimation is presented in Fig. 7. The effective state probability curves after processing are quite similar to original results with effective state number in most of the time no more than 2 , and thus take both state transition continuity and computational efficiency into consideration. The periodic dominant state sequence is in good order and clearly expressed the subject's gait pattern.

After applying the FSM method in the gait analysis of several healthy subjects, the state transition graph for normal walking pattern could be summarized as Fig. 8(a). There are 


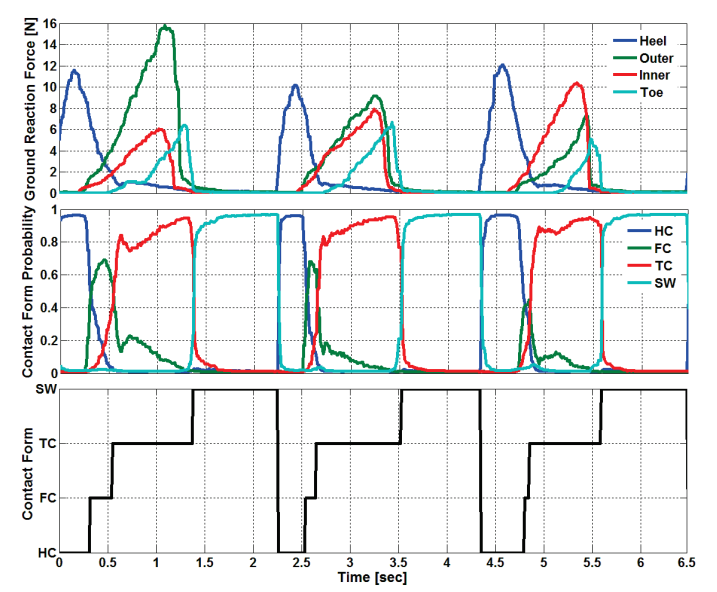

Figure 6. Contact form detection in level ground gait

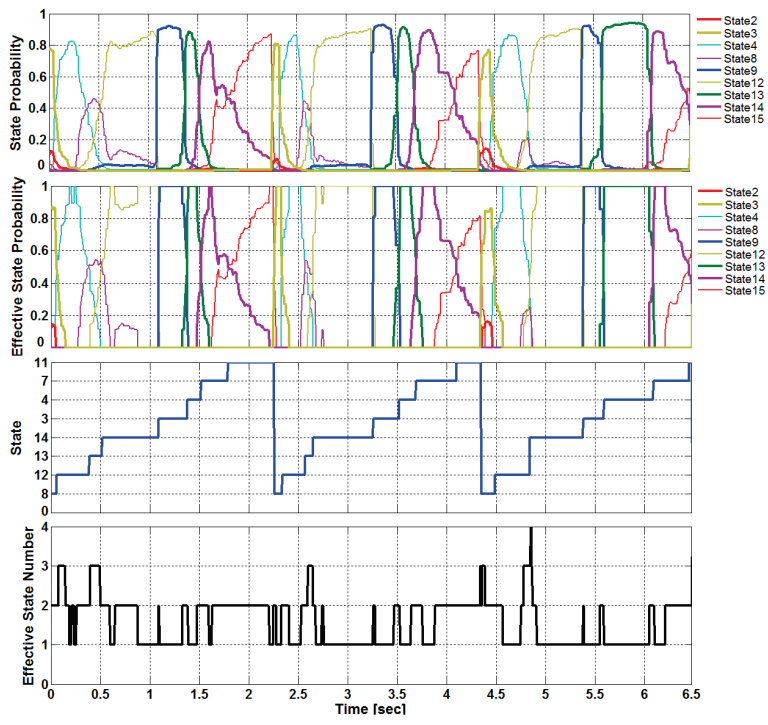

Figure 7. State estimation results in level ground gait

8 states in a gait cycle representing movement of both legs, with start and stop indicated by red and blue arcs. It is worth noting that the state sequence in normal gait cycle may not be unique. When considering the FSM description of disordered gait patterns, we can find from experimental data that two single full support states (8 and 14) always exist in the gait cycle. Based on this observation, all possible state transitions within one gait cycle that follow heel contact ground first principle can be summarized in Fig. 8(b). There are always 8 states in one gait cycle, and the differences in gait patterns exist in FSM state sequences and durations of each state. And these could be used for gait pattern estimation and reference joint angle generation later on in assistive control design.

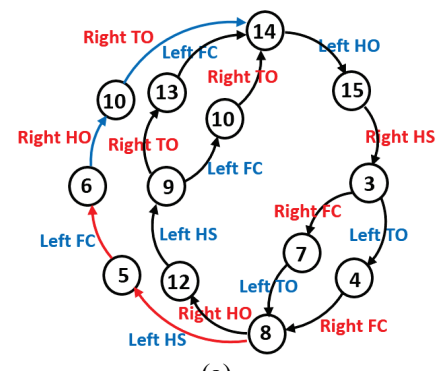

(a)

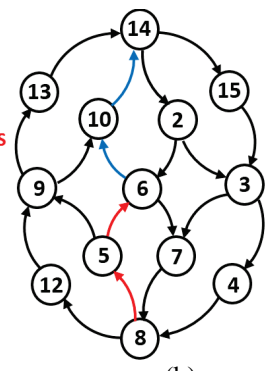

(b)
Figure 8. Level ground gait FSM. (a) Normal pattern, (b) possible pattern

\section{Online Estimation of Gait Pattern}

Online estimation of the gait pattern is the basis for both collection of healthy gait pattern and design of assistive control for disordered gait pattern. Gait pattern in this research refers to the integration of FSM graph and gait parameters such as gait period, percentage of each state in the gait cycle, and knee motion ranges.

1) Gait period: Basically we assume that gait period does not change suddenly during the motions. Therefore, exponential moving average algorithms can be used to estimate gait period by (7), where parameter $\alpha=0.5$ is to let the estimations rely more on recent values. Here, $k$ stands for the $k^{\text {th }}$ gait cycle, $T$ and $T_{\text {est }}$ are the actual and estimated gait period.

$$
T_{e s t, k+1}=\alpha T_{k}+(1-\alpha) T_{e s t, k}, k \geq 0
$$

2) State percentages: Percentage of each state dominated in specific gait pattern is assumed to be constant. State percentages are estimated by cumulative moving average method in (8), with $\lambda_{e s t, k}^{(i)}$ and $t_{k}^{(i)}$ stand for percentage and duration estimation of state $i$ in $k^{\text {th }}$ gait cycle.

$$
\lambda_{e s t, k+1}^{(i)}=\frac{k}{k+1} \lambda_{e s t, k}^{(i)}+\frac{1}{k+1} \cdot \frac{t_{k}^{(i)}}{T_{e s t, k}}, k \geq 0
$$

3) Knee motion range: Knee joint motion range such as maximum and minimum of knee angles, velocities during gait are also collected. Similar to state percentages, cumulative moving average methods are used for estimation of these parameters from gait data.

\section{Assistive Control Design}

The objective of AKB control design is to provide assistance to support the wearer in level ground gait. The generally assistive functions of Powered Lower Limb Orthosis (PLLO) could be explained as follows [10]:

(1) Reference motion pattern: provide appropriate output joint angles similar to normal gait pattern performed by healthy person;

(2) Torque assistance: provide external torque/force in actuating human lower limb or damping function in specific periods of gait cycle.

The general control architecture of Human-PLLO system is shown in Fig. 9, including human body part, orthosis part and assistive controller part. In the orthosis and controller parts, reference joint angle $\theta_{r}$ or reference torque $\tau_{r}$ is obtained according to specific assistive function defined. Then the reference input goes through motion controller to give out control voltage $u_{m}$ to drive the actuator to generate torque $\tau_{m}$ to support human gait.

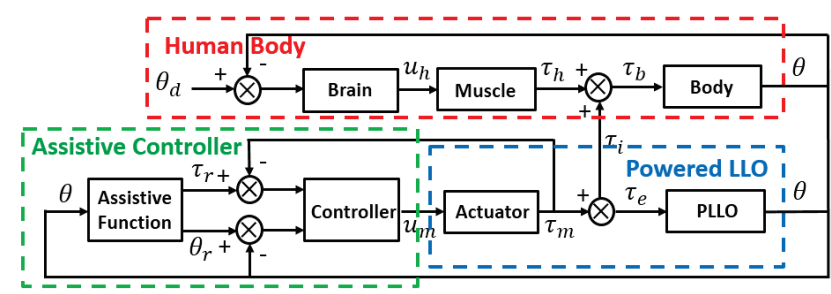

Figure 9. General control architecture of Human-PLLO system 


\section{A. Reference Pattern Generation}

Since the target users of AKB are the elderly or patients that have certain disordered mobility, adaptive, comfortable and safe gait assistance is needed to correct the disordered pattern and approach the normal pattern during rehabilitation. Thus, gait patterns of healthy people were first collected and processed as the normal pattern. And the reference gait pattern provided was online modified based on both the actual gait pattern under control and the normal pattern.

In order to generate reference joint angles that are similar to normal pattern, the joint angle curves in each gait cycle are divided into several phases $[11,12]$. Here phases refer to different parts of the curve with significant differences in slopes. Then from the study of level ground gait data, state sequence could be approximately mapped to 4 phases in knee angle curve as shown in Fig. 10. The knee angle in each phase was generated by linear or quadratic interpolation method based on estimated gait parameters to approximate normal pattern.

The actual gait period $T$, state percentage $\lambda^{(i)}$ for each state $i$ and knee angle maxima $\Theta$ are used to calculate the reference joint angle. The duration estimation of each state is calculated by (9), then by state-phase mapping relationship we could get the duration estimation for phase $j$ as $t p_{e s t}^{(j)}$. Linear interpolations are used in phases 1 and 2 , and the magnitudes of constant knee angular velocity is calculated by (10). And quadratic interpolations in phases 3 and 4 assume that the knee angular accelerations are constant in magnitude calculated by (11). Reference knee angle and velocity calculated should go through boundary checking with upper and lower limits set according to knee motion parameters of normal gait.

$$
\begin{gathered}
t_{e s t, k}^{(i)}=\lambda_{e s t, k}^{(i)} \cdot T_{e s t, k}, k \geq 0 \\
|\omega|=\frac{2 \Theta_{1}}{t p_{e s t}^{(j)}}, j=1,2 \\
|\beta|=\frac{4 \Theta_{2}}{\left(t p_{e s t}^{(j)}\right)^{2}}, j=3,4
\end{gathered}
$$

The reference pattern generation result for a healthy subject without control is given in Fig. 11. It shows that the

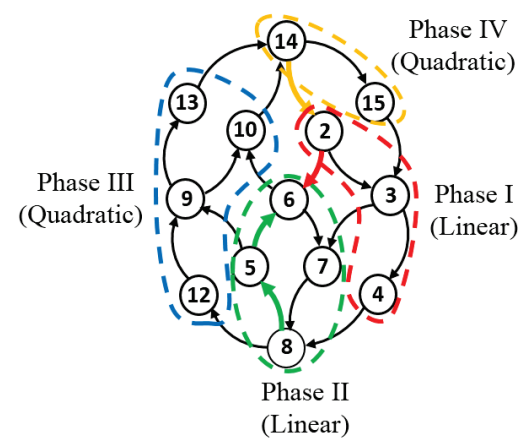

Figure 10. Phase division of level ground gait FSM graph

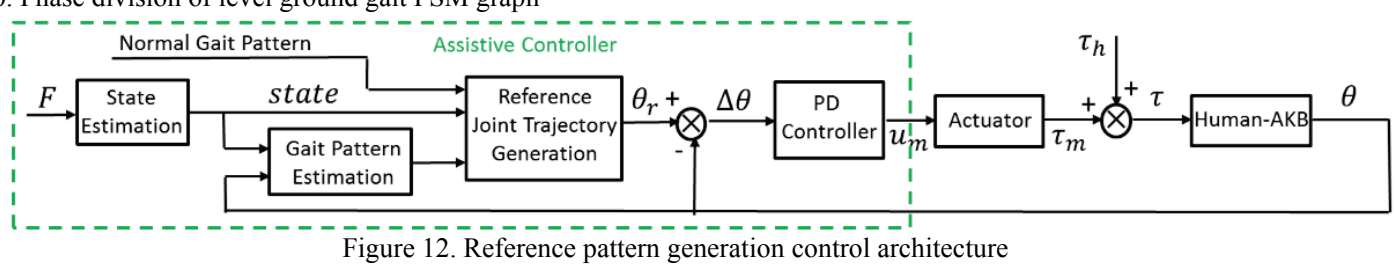

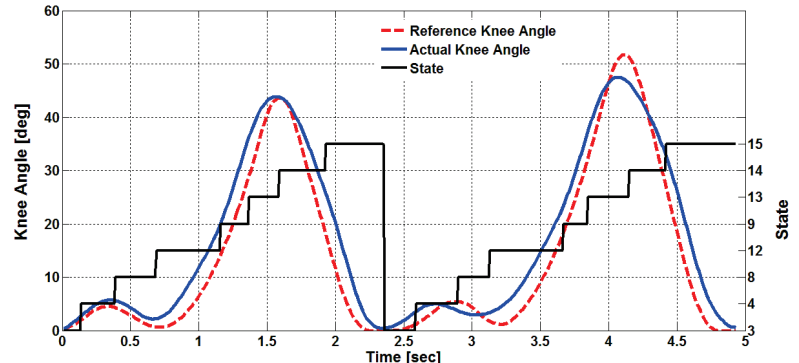

Figure 11. Reference knee angle generation during normal walking

TABLE III. REFERENCE PATTERN MODIFICATION IN GAIT DISORDER

\begin{tabular}{|c|c|c|c|}
\hline Disorder Phase & Phase I & Phase II & Phase III \\
\hline State Transition & $14 \rightarrow 2$ & $2 \rightarrow 6$ & $\begin{array}{c}8 \rightarrow 5 \\
5 \rightarrow 6\end{array}$ \\
\hline Disorder Reason & $\begin{array}{c}\text { Early } \\
\text { right HS }\end{array}$ & $\begin{array}{c}\text { Early } \\
\text { right FC }\end{array}$ & $\begin{array}{c}\text { Late } \\
\text { right HO }\end{array}$ \\
\hline Modification & $\lambda p_{e s t}^{(4)} \uparrow$ & $\lambda p_{\text {est }}^{(1)} \uparrow$ & $\lambda p_{\text {est }}^{(2)} \downarrow$ \\
\hline
\end{tabular}

state sequence could be well mapped to different phases, and generated reference knee angle curve is similar to normal case under healthy gait pattern.

In assistive control for gait rehabilitation, the normal pattern is set as the initial reference gait pattern. If the gait pattern is disordered under control, the reference joint angle based on disordered gait pattern should be modified so as to adjust the gait in the next step. When the subject keeps heel contact ground first in each step, modification of reference joint angle generation for all possible disordered gait patterns should follow those as given in Table III. When disordered state transitions occur, the estimations of phase percentages in gait cycle are modified based on the analysis of specific disorder reasons. Thus, the modified reference could better guide the subject to perform normal walking.

\section{B. AKB Control Architecture}

Based on gait analysis and reference pattern generation methods proposed above, the assistive control scheme of $\mathrm{AKB}$ is shown in Fig. 12. From the sensor input, state is detected and gait parameters are estimated, and then reference pattern is generated as an integration of initial normal pattern and the actual pattern under control. The above are conducted in the remote PC. After that reference knee angle and angular velocity will go through PD controller for actuator control by the embedded controller. Since in each state human body configuration and equivalent dynamic model are different, we have designed variable $\mathrm{P}$ and $\mathrm{D}$ gains tuned from tests for each state.

The low-level control program, shown in Fig. 13, is running at $250 \mathrm{~Hz}$ on embedded controller for data transferring and PD control. Safety checking is performed before the actuator control output. If abnormal situation, e.g. out of working angle, over current occurs, the motor would stop to function automatically to prevent injury. 


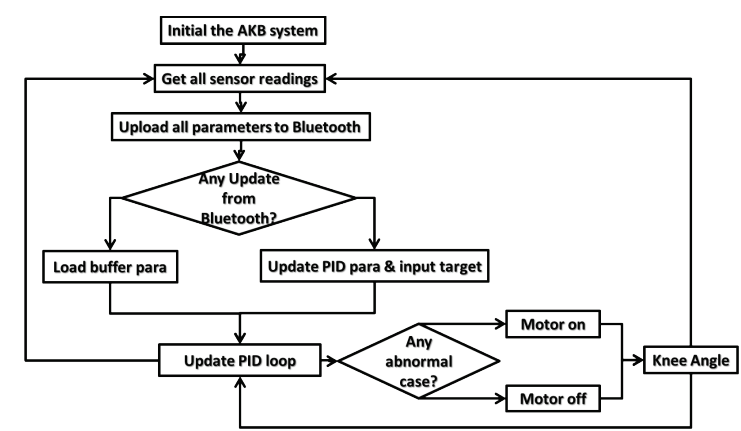

Figure 13. Flow chart of the embedded low-level control program

\section{EXPERIMENTS}

Preliminary experiments were conducted to verify the feasibility of assistive control methods proposed. For safety consideration, before clinical trials we would like to have healthy subjects simulating certain limited mobility to perform the control experiments first. Therefore, in the experiment part, two healthy subjects were asked to walk on the level ground with assistance from AKB in right legs. The first subject was asked to perform a slightly different gait pattern from normal pattern, and the second subject was asked to simulate having muscle weakness around knee.

Experimental results of three continuous gait cycles during the motion of both subjects including state transitions, reference knee angles and actual knee angles under control are shown in Fig. 14. For both subjects with support from AKB the states detected are in good sequence in accordance with the normal pattern, the reference knee curves generated are similar to normal pattern, and the actual knee angles could follow the references quite close with slight lagging about 0.1 second. These show that AKB under control has the ability to correct slight gait disorder to a normal pattern.

The assistive functions could be further investigated through actuator torque curves. For the $1^{\text {st }}$ subject shown in

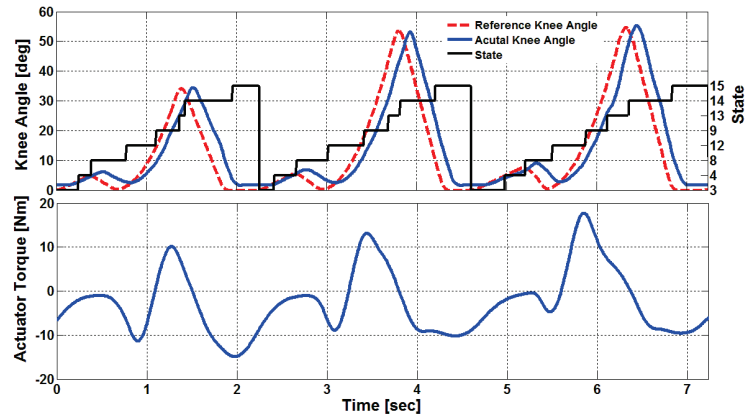

(a)

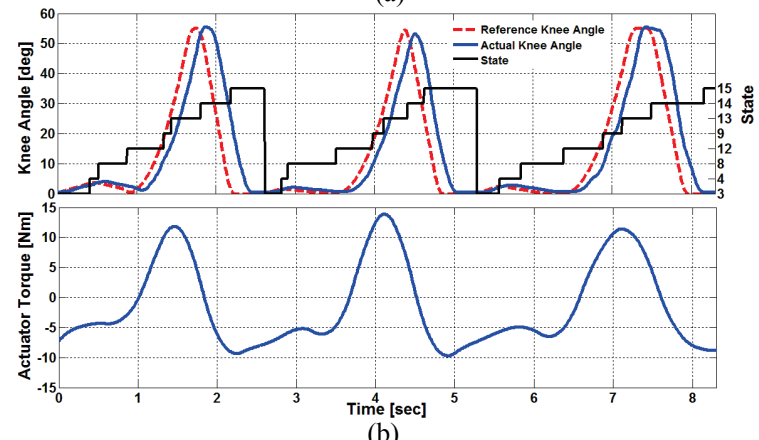

(b)

Figure 14. Experimental results of two subjects under control
Fig. 14(a), a continuous negative torque about $5 \mathrm{Nm}$ in the beginning of stance helped support the body weight, positive torque found mainly in the terminal stance, pre-swing and initial swing stages of the walking cycle with maximal torque about $10 \mathrm{Nm}$ helped in knee flexion to move forward in the end of stance phases, and negative torque followed up to help actuate the knee extension in the swing phase. For the $2^{\text {nd }}$ subject shown in Fig. 14(b), the assistive functions from AKB are similar, but the negative torque was larger in the knee flexion to actuate the shank to follow the designed trajectory, and also negative torque increased in the mid-stance to better support the body weight.

\section{CONCLUSION}

In this study, a light-weight and safe Powered Knee Orthosis was developed to provide assistance in human gait for the elderly and patients with slight mobility problems in their knee joints. With FSM based gait analysis method, individual gait pattern was estimated online. Motion pattern parameters were used to generate reference pattern in assistive control. From the experimental results, AKB controlled by the proposed methods can provide normal reference pattern and proper assistive torque to support human gait. In the near future, the whole system will go through clinical trials to test on patients.

\section{ACKNOWLEDGMENT}

This work is supported by the Start-up Support Scheme, Shenzhen Research Institute, The Chinese University of Hong Kong.

\section{REFERENCES}

[1] K. Suzuki, "Intention-based walking support for paraplegia patients with Robot Suit HAL," Advanced Robotics, vol. 2, no. 12, pp. 1441-1469, 2007.

[2] R. J. Farris, "Preliminary evaluation of a powered lower limb orthosis to aid walking in paraplegic individuals," IEEE Transactions on Neural Systems and Rehabilitation Engineering, vol. 19, no. 6, pp. 652-659, 2011.

[3] A. Goffer, "Gait-locomotor apparatus," U.S. Patent 7153 242, December 26, 2006.

[4] Ekso Bionics. http://www.eksobionics.com/ekso.

[5] R. L. Norton, "Belt and Chain Drvies," in Kinematics and Dynamics of Machinery, $1^{\text {st }}$ Edition in SI Units, ch.9, New York: McGraw Hill, 2009, pp.473-474.

[6] H. Kazerooni, "The Berkeley lower extremity exoskeleton," Journal of Dynamic System, Measurement, and Control, Transactions of $A S M E$, vol. 128, pp. 14-25, 2006.

[7] S. R. Edgar, "Wearable shoe-based device for rehabilitation of stroke patients," Proc. of the $32^{\text {st }}$ Annual Int'l Conf. of the IEEE EMBS, Buenos Aires, Argentina, Sep. 2010, pp. 3772-3775.

[8] K. Kong, "Smooth and continuous human gait phase detection based on foot pressure patterns," Proc. of the 2008 IEEE Int'l Conf. on Robotics and Automation, Pasadena, CA, May. 2008, pp. 3678-3683.

[9] A. Alvarez-Alvarez, "Human gait modeling using a genetic fuzzy finite state machine," IEEE Trans. Fuzzy System, vol. 20, no. 2, pp. 205-223, Apr. 2012.

[10] I. Diaz, "Lower-limb robotic rehabilitation: literature review and challenges," Journal of Robotics, vol. 2011, pp. 1-11, 2011.

[11] A. Tsukahara, "Gait support for complete spinal cord injury patient by synchronized leg-swing with HAL," Proc. of the 2011 IEEE/RSJ Int'l Conf. on Intelligent Robots and Systems, San Francisco, CA, Sep. 2011, pp. 1737-1742.

[12] H. Kawamoto, "Development of single leg version of HAL for hemiplegia," Proc. of the $31^{\text {st }}$ Annual Int'l Conf. of the IEEE EMBS, Minneapolis, Minnesota, Sep. 2009, pp. 5038-5043. 